\title{
Escala de Urgência com o Tempo e Ocupação Constante: Adaptação, Validade e Versão Reduzida
}

\author{
Time Urgency and Perpetual Activation Scale: Adaptation, Validity \\ and a Short-Version
}

\author{
Renan Benigno Saraiva, Camila Azevedo Gastal, Gabriela Campelo \& Fabio Iglesias* \\ Universidade de Brasília, Brasília, DF, Brasil
}

\begin{abstract}
Resumo
Cada indivíduo possui um repertório de estratégias e maneiras de lidar com o tempo, diferenciando-se na urgência com que realiza suas tarefas e na quantidade de compromissos a que se submete. Alguns instrumentos foram propostos para acessar tais diferenças individuais em relação ao tempo, destacando-se a Time Urgency and Perpetual Activation Scale (TUPA), que acessa níveis de urgência com o tempo e ativação constante. Neste trabalho são descritas a adaptação e as evidências de validade da TUPA para o contexto brasileiro, assim como a proposta de uma versão reduzida. A escala traduzida foi submetida por meio de formulários online a 395 participantes $(71,4 \%$ mulheres), com idades de 17 a 64 anos $(M=25,36, D P=8,65)$. Análises fatoriais exploratórias e confirmatórias apontaram uma nova estrutura bifatorial, com boas propriedades psicométricas, envolvendo um fator de urgência com o tempo e outro relacionado a ocupações, nomeada como Escala de Urgência com o Tempo e Ocupação Constante (UTOC). Conclui-se que a UTOC apresentou boas evidências de validade de construto, incluindo validade convergente com medidas robustas de ansiedade e estresse. Palavras-chave: Urgência com o tempo, ocupação constante, ansiedade.
\end{abstract}

\begin{abstract}
Each individual has a repertoire of strategies for dealing with time, differentiating themselves in terms of levels of urgency when performing tasks and the number of appointments they are committed to. A few instruments have been developed to access these individual differences concerning time, notably the Time Urgency and Perpetual Activation Scale (TUPA), which measures levels of time urgency and perceptual activation. This paper describes a translated and adapted TUPA for the Brazilian context, and proposes its short-version. The translated scale was submitted as part of an online survey to 395 participants $(71.4 \%$ female), ranging in age from 17 to 64 years $(M=25.36, S D$ $=8.65$ ). Exploratory and confirmatory factor analyses suggested a new bifactorial solution with good psychometric properties, involving a time urgency dimension and another related to occupations, named Time Urgency and Constant Ocupation Scale (UTOC). The UTOC showed good evidence of construct validity, as well as appropriate levels of convergent validity with robust measures of anxiety and stress.

Keywords: Time urgency, perpetual activation, anxiety.
\end{abstract}

Atitudes, hábitos e decisões são influenciados pela maneira como percebemos e pensamos acerca do nosso tempo, tanto em níveis de análise sociais quanto individuais. O ritmo de vida em grandes cidades, por exemplo, está diretamente associado à incidência de doenças cardía-

\footnotetext{
* Endereço para correspondência: Universidade de Brasília, Instituto Central de Ciências Sul, Laboratório de Psicologia Social, sala A1-116, Brasília, DF, Brasil 70900-100. E-mail: iglesias@unb.br

Agradecimentos: Os autores agradecem à Fundação de Apoio a Pesquisa do Distrito Federal (FAPDF) pelo apoio na forma de equipamentos e à Coordenação de Aperfeiçoamento de Pessoal de Nível Superior (CAPES) e ao Conselho Nacional de Desenvolvimento Científico e Tecnológico ( $\mathrm{CNPq}$ ) pelo apoio na forma de bolsas de pesquisa.
}

cas, à quantidade de fumantes e à sensação de bem-estar subjetivo (Levine \& Norenzayan, 1999). Outras diferenças individuais relacionadas ao tempo possuem uma série de repercussões em aspectos cognitivos e comportamentais, desde comportamentos no trânsito (Zimbardo, Keough, \& Boyd, 1997) até sensações de felicidade (DeVoe \& House, 2012). Diversas ferramentas metodológicas foram elaboradas para acessar de que forma as pessoas diferenciam-se em suas percepções sociais e psicológicas do tempo. Porém, poucos instrumentos que mensuram tais fenômenos foram produzidos ou adaptados para o contexto brasileiro (Leite \& Pasquali, 2008; Milfont, Andrade, Pessoa, \& Belo, 2008). O objetivo geral deste trabalho foi adaptar e buscar evidências de validade no Brasil para um instrumento relacionado a variáveis temporais, focado principalmente 
na urgência com o tempo. Adicionalmente, o trabalho teve o objetivo de propor uma versão reduzida do instrumento.

$\mathrm{O}$ interesse científico por diferenças individuais relacionadas ao tempo se desenvolveu mais claramente pelos estudos sobre o chamado padrão de comportamento Tipo A: um conjunto de ações e hábitos que configuram um ritmo de vida acelerado, relacionado principalmente à propensão a doenças coronárias (Friedman \& Rosenman, 1959; Sirri et al., 2012). Os fatores que compõem o padrão de comportamento Tipo A envolvem níveis elevados de competitividade, impaciência, preocupação com prazos, urgência com o tempo e ativação constante. Dentre eles, a urgência com o tempo recebeu um destaque maior, tanto pela sua operacionalização conceitual quanto pelo seu maior valor preditivo (Fernandes, Hatfield, \& Soames Job, 2010; Gastorf, 1980; Menon, Narayanan, \& Spector, 2013; Wright, McCurdy, \& Rogoll, 1992; Yarnold \& Grimm, 1982; Yarnold \& Mueser, 1984). Trata-se, portanto, de construto altamente relevante, tanto para os esforços de ciência básica quanto aplicada a diversas áreas.

\section{Urgência com o Tempo}

A urgência com o tempo costumava ser definida como um construto unidimensional, relacionado somente à impaciência (Landy, Rastegary, Thayer, \& Colvin, 1991), mas passou a ser interpretada como multidimensional, com fatores que envolvem: consciência do tempo, hábitos alimentares, planejamento, energia, padrões de fala e controle de prazos (Conte, Landy, \& Mathieu, 1995), além de realização de muitas tarefas ao mesmo tempo, pontualidade, velocidade e pressão temporal (Edwards, Baglioni, \& Cooper, 1990; Kohler, 1991; Landy et al., 1991). As investigações envolvendo esse construto tomaram grandes proporções, alcançando diversos campos de aplicação. No âmbito da saúde, por exemplo, é possível identificar que indivíduos com maior urgência com o tempo sofrem de mais enfermidades de sono, digestão, respiração, motoras e dor (Conte, Mathieu, \& Landy, 1998; Shi et al., 2013). Tais evidências expõem que a urgência com o tempo é um notável fator de risco ao bem-estar fisiológico e somático de indivíduos, o que ressalta a importância de medidas que permitam acessar níveis patogênicos de tal condição, assim como fornecer recursos para a sua identificação precoce.

Variáveis relacionadas ao tempo também se destacam nos contextos organizacionais e de trabalho. A urgência com o tempo é comumente associada com um desempenho prejudicado em diversas tarefas e atividades de resolução de problemas (Friend, 1982; Glass, Snyder, \& Hollis, 1974). Tal piora é atenuada quando as características do indivíduo e a natureza da tarefa são incongruentes, de forma que indivíduos mais preocupados com seu tempo têm pior desempenho em tarefas que não demandam urgência (Greenberg, 2002). Parte destas variações no desempenho podem ser atribuídas a níveis elevados de estresse e ansiedade provenientes da urgência com o tempo, principalmente em contextos laborais que envolvem o desempenho de atividades repetitivas e com pressão temporal (Conte, Schwenneker, Dew, \& Romano, 2001; Menon et al., 2013). Outros estudos que complementam essas evidências envolvem o gerenciamento do tempo, um construto que incorpora algumas dimensões da urgência com o tempo, mas que se distingue quanto à eficiência ao planejar e organizar o próprio tempo (ver Claessens, van Eerde, Rute, \& Roe, 2007, para uma revisão). Indivíduos com melhor gerenciamento de tempo possuem mais satisfação no trabalho, percepção de controle do tempo e melhor desempenho acadêmico (Britton \& Tesser, 1991; Macan, Shahani, Dipboye, \& Phillips, 1990).

Esse conjunto de evidências confere grande valor preditivo a variáveis relacionadas ao tempo, reforçando sua relevância na compreensão de uma ampla variedade de comportamentos e atitudes. O avanço nessa temática depende de diversos instrumentos de mensuração, elaborados com o intuito de melhor compreender como indivíduos percebem e lidam com seu próprio tempo. Sendo assim, muito foi feito para refinar a conceituação de tais traços disposicionais, assim como para elaborar instrumentos que permitissem mensurá-los de forma objetiva.

\section{Medidas de Diferenças Individuais em Relação ao Tempo}

Alguns dos primeiros instrumentos elaborados para mensurar variáveis relacionadas ao tempo tinham o intuito de discriminar indivíduos Tipo A de indivíduos Tipo B, principalmente por meio de entrevistas (Rosenman et al., 1966) e questionários (Jenkins, Zyzanski, \& Rosenman, 1979; Krantz, Glass, \& Snyder, 1974). O uso de tais medidas foi abandonado ao longo do tempo, uma vez que surgiram dificuldades de caráter psicométrico em acessar variáveis tão amplas e multidimensionais (Edwards et al., 1990). Dessa forma, medidas mais recentes tenderam a focar em um número reduzido e mais específico de componentes da relação individual com o tempo. Como exemplo, é possível citar instrumentos que focam em aspectos da percepção de padrões de tempo (Usunier \& Valette-Florence, 1994), preferência por quantidade de tarefas realizadas ao mesmo tempo (Bluedorn, Kaufman, \& Lane, 1992) e gerenciamento do tempo (Macan et al., 1990). Ainda dentro da proposta de medir características específicas e disposicionais da relação com o tempo, Wright et al. (1992) desenvolveram a Time Urgency and Perpetual Activation Scale, que é hoje um dos instrumentos mais utilizados nas pesquisas que envolvem especificamente o construto de urgência temporal.

\section{Time Urgency and Perpetual Activation Scale - TUPA}

Diversos estudos têm utilizado a TUPA para avaliar de que forma a urgência com o tempo está relacionada a indicadores de qualidade de vida, saúde e outros construtos. No âmbito da saúde, por exemplo, o papel preditivo da TUPA foi testado na incidência de doenças crônicas coronárias, câncer e ameaças comuns à maioria da população (Amelang, 1997; Wright et al., 1995; Yousfi, Matthews, Amelang, \& Schmidt-Rathjens, 2004). Ela foi formulada a 
Saraiva, R. B., Gastal, C. A., Campelo, G. \& Iglesias, F. (2015). Escala de Urgência com o Tempo e Ocupação Constante: Adaptação, Validade e Versão Reduzida.

partir do relato de pacientes com doenças coronárias sobre as atividades cotidianas que realizavam, de forma que 73 itens foram extraídos desses exemplos de atividades (não relacionadas a hospitalização ou qualquer consequência de doença coronária). Em seguida, os itens foram aplicados a indivíduos já diagnosticados como Tipo A ou B, juntamente com uma entrevista para mensurar urgência com o tempo e ativação constante, entre outros subcomponentes do padrão Tipo A (Wright \& Schmidt-Walker, 1990). Dos 73 itens iniciais, apenas 47 se correlacionaram com a urgência com o tempo e/ou ativação constante, compondo a TUPA. Para chegar à versão final do instrumento, ainda foram adicionados 25 itens distratores à escala, de forma que a escala final é composta por 72 itens.

Considerando a relevância do instrumento, esta pesquisa teve por objetivo adaptar para o contexto brasileiro e buscar evidências de validade da TUPA, assim como propor uma versão reduzida da escala. Espera-se suprir parte da necessidade por instrumentos em língua portuguesa que acessem indicadores da relação individual com o tempo, capazes de mensurar dimensões específicas tais como níveis de ativação e urgência com o tempo.

\section{Método}

\section{Participantes}

A amostra incluiu 395 participantes voluntários com idades de 17 a 64 anos $(M=25,36, D P=8,65)$, sendo $71,4 \%$ mulheres. Os participantes foram recrutados por meio de redes sociais online de universidades. A escolaridade variou de ensino médio completo a ensino superior completo: 4,6\% tinha até o ensino médio completo, $61,3 \%$ estava cursando o ensino superior e $28,4 \%$ tinha o ensino superior completo.

\section{Instrumentos}

Time Urgency and Perpetual Activation Scale (TUPA). Traduziu-se o questionário da versão original em inglês (Wright et al., 1992), utilizando o método de retrotradução (van de Vijver \& Leung, 2011). A versão original foi traduzida do inglês para o português pelos autores. A escala traduzida foi posteriormente retraduzida para o inglês por um indivíduo fluente em ambas as línguas. Em seguida o instrumento original foi comparado com a versão retraduzida para o inglês pelos próprios autores, fluentes em ambos os idiomas, sendo realizados ajustes na tradução em português quando necessário. A nomeação das categorias de resposta foi adaptada para 1 - Extremamente falso para mim; 2 - Moderadamente falso para mim; 3 - Levemente falso para mim; 4 - Levemente verdadeiro para mim; 5 Moderadamente verdadeiro para mim; e 6 - Extremamente verdadeiro para mim. A escala original é composta por 47 itens (somados a 25 distratores), organizados da seguinte forma: 7 itens relacionados unicamente à urgência com o tempo; 9 itens unicamente relacionados à ativação constante; e um fator global que envolve todos os 47 itens. Durante o processo de tradução optou-se por remover o item 14 da escala ("When I was in school, I held two or more offices in groups such as student council, glee club, 4-H club, sorority, or team"). A eventual adaptação desse item, além de se distanciar do sentido original, restringiria a aplicação da escala, uma vez que os correspondentes de tais atividades no contexto brasileiro não são comuns à toda a população. Sendo assim, a versão final do instrumento traduzido contou com 71 itens.

Inventário de Ansiedade Traço-Estado (IDATE). Desenvolvido por Spielberger, Gorsuch e Lushene (1970), o IDATE mede níveis de ansiedade, tanto em termos de estado quanto de traço. A ansiedade-estado é uma condição temporária que engloba sensações de tensão, stress, nervosismo e apreensão. Já a ansiedade-traço refere-se a um nível mais estável de comportamentos do indivíduo, verificando diferenças individuais em relação à tendência de reagir a situações percebidas como ameaçadoras. Foi utilizada apenas a versão ansiedade-traço adaptada com indícios de validade para o contexto brasileiro (Biaggio \& Natalício, 1979; Fioravanti, Santos, Cruz, \& Landeira-Fernandez, 2006). Optou-se por utilizar a versão ansiedade-traço por ser uma medida de diferença individual assim como a TUPA, de forma que uma medida situacional (ansiedade-estado) seria inadequada. Essa versão possui 20 itens, separados em dois fatores: um que visa acessar o traço de ansiedade dos respondentes $(\alpha=0,88$; Ex: "Sinto que as dificuldades estão se acumulando de tal forma que não as consigo resolver"), com 13 itens, e outros 7 itens que indicam a ausência desse traço de ansiedade $(\alpha=0,85$; Ex: "Sou calmo, ponderado e senhor de mim mesmo"). Os itens deviam ser respondidos utilizando-se uma escala que varia de 1- Quase nunca até 4 - Quase sempre.

Escala de Estresse Percebido - Perceived Stress Scale (PSS). Originalmente elaborada por Cohen, Karmack e Mermelsteinm (1983), a escala visa medir o grau no qual indivíduos percebem situações como estressantes. A versão com evidências de validade para o contexto brasileiro utilizada neste estudo (Luft, Sanches, Mazo, \& Andrade, 2007) possui dois fatores: Estresse Percebido ( $\alpha=0,82$; Ex: "Você tem se sentido incapaz de controlar as coisas importantes em sua vida?"), com 7 itens; e Coping Percebido ( $\alpha$ $=0,87$; Ex: "Você tem conseguido controlar as irritações em sua vida?") também com 7 itens. $\mathrm{O}$ instrumento é apresentado numa escala de 0 - Nunca a 4 - Sempre.

\section{Procedimentos}

A coleta de dados foi realizada por meio de um survey online. Os participantes foram convidados por e-mail e em anúncios divulgados em redes sociais. Os anúncios continham instruções e um link para o questionário, com um termo de autorização, conforme todas as recomendações éticas aplicáveis e em conformidade com o comitê de ética em pesquisa institucional que autorizou a condução da pesquisa. Foram apresentados aos participantes a TUPA, o Inventário de Ansiedade-Traço, e a Escala de Estresse Percebido, nesta ordem. Instruções específicas foram destacadas a cada página, alertando para as diferenças no 
preenchimento dos instrumentos. Ao final, os participantes foram requisitados a informar seu sexo, idade e nível de escolaridade. O tempo médio de resposta ao survey foi de 20 minutos.

\section{Análise dos Dados}

Inicialmente a estrutura fatorial da TUPA foi verificada por meio de análises fatoriais exploratórias por eixos principais, com método de rotação oblíqua, tendo em vista que os fatores são teoricamente correlacionados. A consistência interna dos fatores originais da TUPA e dos obtidos na análise fatorial exploratória foi verificada por meio do Alfa de Cronbach. Em seguida, a adequação de cada um dos modelos obtidos foi testada por meio de modelagem de equações estruturais, contrastando o modelo trifatorial da escala original com um modelo bifatorial obtido na análise fatorial exploratória. Também foi avaliada a possibilidade de utilizar uma versão reduzida da escala utilizando os itens com maior carga fatorial. Por fim, foram verificadas evidências de validade convergente via correlações entre os escores da TUPA, da PSS e da IDATE.

Optou-se por não incluir nas análises seis itens da TUPA, por se referirem a comportamentos tipicamente relacionados ao trânsito. Tal decisão foi embasada na caracterização da amostra obtida, que possibilita que muitos dos participantes não experienciem diretamente tais situações como motoristas (se menores de 18 anos). Além disso, a retirada de tais itens possibilita que o instrumento seja aplicável em diversos contextos. O banco de dados foi submetido a procedimentos de análise exploratória, para verificação de pressupostos e adequação ao modelo linear geral (Tabachnick \& Fidell, 2013). A quantidade de dados omissos não superou $5 \%$ da amostra para cada variável, mas ainda assim tais casos foram substituídos usando o algoritmo EM (expectation maximization). Não foram detectados casos extremos univariados ou multivariados, avaliados por meio da distância de Mahalanobis. Também não foram encontrados problemas relativos à singularidade e multicolinearidade dos dados.

Quanto à distribuição dos itens, houve assimetrias positivas que variaram de 0,004 a 1,366 , enquanto as assimetrias negativas variaram de $-0,03$ a $-0,814$. Os valores de achatamento variaram de $-0,133$ a 0,642 . O coeficiente de normalidade multivariada (Mardia, 1971) também indicou uma distribuição não normal dos itens no banco de dados. Entretanto, optou-se por utilizar o algoritmo de máxima verossimilhança (ML) na análise fatorial confirmatória (AFC) para estimação dos parâmetros, considerando que a anormalidade da distribuição nos itens não foi tão elevada.

Os índices avaliados para testes de ajuste do modelo nas análises fatoriais confirmatórias foram o $\mathrm{c}^{2} ; \mathrm{CFI}(\mathrm{Com}$ parative Fit Index); RMSEA (Root Mean Square Error of Aproximation); NFI (Normed Fit Index) e GFI (Goodness-of-fit-Index), escolhidos de acordo com a literatura da área (Boomsma, 2000; Byrne, 2010; McDonald \& Ho, 2002). Os valores de cada índice adotados como critério de ajuste foram: CFI superior a 0,90; RMSEA próximo ou inferior a 0,08 ; GFI superior a 0,90 ; e NFI superior a 0,90 .

\section{Resultados}

Inicialmente verificou-se que a amostra é adequada à análise fatorial exploratória $(\mathrm{KMO}=0,84)$. Um exame dos percentuais da variância explicada, do gráfico de sedimentação e dos autovalores sugeriu a extração de dois ou três fatores da TUPA. De acordo com o obtido nas primeiras análises dos eixos principais, procedeu-se com a extração de dois fatores. Foram definidos o método de rotação oblíqua e critério de exclusão para itens com carga fatorial inferior a 0,30 . Os itens que compõem cada fator, com suas respectivas cargas fatoriais são apresentados na Tabela 1. O primeiro fator integrou 21 itens correspondentes à Urgência com o Tempo $(\alpha=0,83 ; M=3,44$; $D P=0,79)$. O segundo fator integrou 10 itens associados à Ocupação Constante $(\alpha=0,72 ; M=3,51 ; D P=0,89)$. Procedeu-se com a extração de três fatores, a fim de avaliar outra possível solução que seguisse a composição original da escala. Foi utilizado novamente o método de rotação oblíqua e exclusão de cargas menores que 0,30. Porém, a interpretação conceitual dos fatores foi comprometida, uma vez que os itens dos diferentes fatores guardaram muitas semelhanças. Sendo assim, optou-se por utilizar somente a solução bifatorial da análise fatorial exploratória.

Foi testada também uma versão reduzida da escala, buscando-se encontrar uma solução unifatorial. Foram adotados apenas itens com cargas fatoriais maiores que 0,40 , com o intuito de selecionar os mais representativos. Atingiram o critério de inclusão 23 itens, formando um único fator nomeado Urgência com o Tempo $(\alpha=0,85)$. Os itens da versão reduzida, com suas respectivas cargas fatoriais, também são apresentados na Tabela 1.

Os fatores obtidos por meio da análise fatorial exploratória apresentaram uma composição distinta da sugerida pelos autores originais da escala. Tanto a solução original quanto a solução bifatorial obtida neste estudo possuem um fator referente à urgência com o tempo, mas os itens que as compõem não são exatamente correspondentes. Também foi encontrado um novo fator, relacionado a níveis de ocupação constante, com itens que também não correspondem aos de ativação perpétua da versão original. Sendo assim, a nova composição da escala é nomeada daqui em diante como Escala de Urgência com o Tempo e Ocupação Constante (UTOC). Em seguida, foram realizadas análises fatoriais confirmatórias com o intuito de identificar qual destes modelos melhor se ajustava aos dados obtidos. As reespecificações realizadas nos modelos TUPA (solução original) e UTOC (solução bifatorial e versão reduzida) foram covariâncias significativas entre erros, não previstas inicialmente. Tais reespecificações só foram feitas entre itens que possuíam alguma semelhança semântica, respeitando recomendações para tal procedimento (Boomsma, 2000; McDonald \& Ho, 2002). Essa análise indicou que o modelo de Urgência com o Tempo e Ocupação Constante (UTOC) possuiu índices satisfatórios $(\mathrm{GFI}=0,90)$. Os índices de adequação de ambos os modelos, assim como a dos reespecificados (com covariâncias entre erros), estão descritos na Tabela 2. 
Saraiva, R. B., Gastal, C. A., Campelo, G. \& Iglesias, F. (2015). Escala de Urgência com o Tempo e Ocupação Constante: Adaptação, Validade e Versão Reduzida.

Tabela 1

Cargas Fatoriais para Cada Item da Solução Bifatorial e da Versão Reduzida da Escala de Urgência com o Tempo e Ocupação Constante (UTOC)

\begin{tabular}{|c|c|c|}
\hline UT & OC & $\begin{array}{c}\text { Versão } \\
\text { Reduzida }\end{array}$ \\
\hline 0,64 & $-0,08$ & 0,52 \\
\hline $\mathbf{0 , 5 8}$ & 0,00 & $\mathbf{0 , 5 3}$ \\
\hline 0,54 & $-0,03$ & $\mathbf{0 , 4 7}$ \\
\hline $\mathbf{0 , 5 3}$ & $-0,08$ & 0,42 \\
\hline $\mathbf{0 , 5 3}$ & $-0,01$ & 0,48 \\
\hline 0,52 & $-0,03$ & 0,46 \\
\hline $\mathbf{0 , 5 0}$ & $-0,06$ & $\mathbf{0 , 4 1}$ \\
\hline $\mathbf{0 , 5 0}$ & 0,00 & 0,46 \\
\hline $\mathbf{0 , 5 0}$ & 0,10 & $\mathbf{0 , 5 3}$ \\
\hline 0,49 & 0,07 & 0,51 \\
\hline 0,49 & $-0,02$ & 0,43 \\
\hline 0,45 & $-0,10$ & - \\
\hline $\mathbf{0 , 4 5}$ & $-0,04$ & - \\
\hline 0,45 & 0,31 & 0,64 \\
\hline 0,45 & 0,09 & $\mathbf{0 , 4 8}$ \\
\hline 0,42 & 0,13 & 0,48 \\
\hline 0,42 & 0,10 & 0,46 \\
\hline $\mathbf{0 , 3 2}$ & $-0,10$ & - \\
\hline $\mathbf{0 , 3 2}$ & 0,24 & 0,47 \\
\hline $\mathbf{0 , 3 0}$ & $-0,01$ & - \\
\hline $\mathbf{0 , 3 0}$ & 0,17 & $\mathbf{0 , 4 0}$ \\
\hline 0,26 & 0,26 & 0,42 \\
\hline$-0,07$ & 0,72 & 0,45 \\
\hline
\end{tabular}

48. Eu acho que portas automáticas abrem muito devagar, e que geralmente tenho que diminuir a velocidade para evitar colidir com elas.

44. Quando preciso ficar sentado, eu manuseio um objeto (como um lápis), faço movimentos com os dedos, movimento meus dentes, ou não fico completamente parado.

68. Quando eu chego cedo para uma reunião, eu fico impaciente esperando que comece.

49. Eu mantenho meus dentes pressionados juntos, sem ranger, mas com o maxilar tenso.

17. É difícil, para mim, sentar para uma longa refeição.

62. Eu posso me sentir inclinado a interromper as pessoas se elas não estão respondendo ou reagindo no sentido que deveriam.

60. Eu me encontro competindo com colegas.

4. Eu odeio errar digitando um número de telefone e ter que começar tudo de novo.

9. Pessoas que eu conheço bem concordam que eu tendo a fazer a maioria das coisas com pressa.

46. As únicas vezes em que me sinto confortável me movendo devagar são quando estou doente.

32. Eu abro coisas de forma rápida e impetuosa, às vezes rasgando caixas ou cartas para abri-las, ao invés de fazer isso tenuemente ou cortando-as gentilmente com um abridor apropriado.

21. Eu sinto que estou ficando para trás ou que as coisas estão me sufocando.

14. As pessoas dizem que eu mastigo comida ou masco chiclete de forma mais vigorosa do que a maioria das pessoas.

31. Eu acho necessário me apressar muito mais do que os meus colegas.

27. Quando estou pegando algo de dentro de um recipiente, eu o faço rapidamente.

10. Eu fico impaciente com pessoas que são capazes de trabalhar em um ritmo mais lento e menos estruturado.

35. É difícil, para mim, ficar sentado terminando uma refeição e conversar depois de terminar a refeição.

15. Às vezes eu subo as escadas dois degraus por vez.

25. Eu fico frustrado quando colegas querem me visitar ou conversar casualmente comigo enquanto estou ocupado.

69. Eu faço careta quando estou me exercitando.

65. Eu pareço planejar que certas tarefas vão tomar menos tempo do que elas eventualmente acabam tomando.

56. Eu sou exigente ou rígido com tecnologias, máquinas ou veículos.

$-0,07$

0,72

0,45 


\begin{tabular}{lccc}
\hline & UT & OC & $\begin{array}{c}\text { Versão } \\
\text { Reduzida }\end{array}$ \\
\hline $\begin{array}{l}\text { 64. As pessoas dizem que eu sou uma pessoa muito ocupada, uma das mais ocu- } \\
\text { padas que elas conhecem. }\end{array}$ & 0,00 & $\mathbf{0 , 7 0}$ & $\mathbf{0 , 5 0}$ \\
66. Eu trabalho e/ou estudo consideravelmente mais do que oito horas por dia & $-0,09$ & $\mathbf{0 , 7 0}$ & $\mathbf{0 , 4 1}$ \\
$\begin{array}{l}\text { 71. Eu me preparo para atividades com antecedência para que eu não desperdice } \\
\text { tempo ou tenha que voltar para pegar coisas que esqueci. }\end{array}$ & $-0,13$ & $\mathbf{0 , 5 1}$ & - \\
$\begin{array}{l}\text { 22. Sou cuidadoso ao realizar tarefas em uma sequência ordenada, para realizá-las } \\
\text { no menor tempo possível. }\end{array}$ & 0,05 & $\mathbf{0 , 4 7}$ & - \\
$\begin{array}{l}\text { 30. Durante um compromisso, eu deixo claro para a outra pessoa que tenho outro } \\
\text { compromisso ou que sou uma pessoa ocupada, assim conduzo nosso encontro } \\
\text { mais objetivamente. }\end{array}$ & 0,14 & $\mathbf{0 , 4 4}$ & $\mathbf{0 , 4 4}$ \\
39. Mais do que uma vez por semana eu levo meu trabalho para casa a noite ou & 0,04 & $\mathbf{0 , 4 1}$ & - \\
estudo materiais relacionados ao trabalho. & & & $\mathbf{0 , 3 6}$ \\
3. Eu agendo atividades o mais próximo possível de meus compromissos para não \\
desperdiçar tempo.
\end{tabular}

Nota. UT - Urgência com o Tempo; OC - Ocupação Constante. Cargas fatoriais em negrito (>s 0,30) sinalizam a composição final dos itens em cada fator e na versão reduzida.

Tabela 2

Índices de Adequação dos Modelos Testados

\begin{tabular}{cccccc}
\hline & $\chi^{2}$ & CFI & RMSEA & NFI & GFI \\
\hline Modelo TUPA & $2202,382(G L=1001)$ & 0,661 & 0,05 & 0,524 & 0,797 \\
Modelo TUPA reespecificado & $0,817(G L=990)$ & 0,722 & 0,05 & 0,573 & 0,817 \\
Modelo UTOC & $1976,912(G L=433)$ & 0,782 & 0,054 & 0,66 & 0,863 \\
Modelo UTOC reespecificado & $660,845(G L=418)$ & 0,892 & 0,038 & 0,757 & 0,902 \\
\hline
\end{tabular}

Notas. CFI - Comparative Fit Index; RMSEA - Root Mean Square Error of Approximation; NFI - Normed Fit Index; GFI Goodness-of-Fit Index.

Por fim foram realizadas análises correlacionais entre os índices da IDATE e PSS, a fim de averiguar a validade convergente da escala adaptada. Foram acrescentados à análise os índices dos fatores originais da TUPA, dos fatores da solução UTOC e do fator da escala reduzida. Os valores de tais correlações, assim como seus níveis de significância são apresentados na Tabela 3.

\section{Discussão}

O objetivo deste estudo consistiu em traduzir e adaptar a Time Urgency and Perpetual Activation Scale (TUPA;
Wright et al., 1992) para o contexto brasileiro, testando suas qualidades psicométricas e reunindo evidências de validade para seu uso. Tanto os fatores originais da escala quanto novas soluções fatoriais foram investigadas, uma vez que especificidades culturais possibilitaram a formulação de novos componentes. Foram observadas algumas divergências entre a composição dos fatores da escala original frente à nova solução fatorial encontrada neste estudo. Sendo assim, desenvolveu-se uma versão brasileira da TUPA, com uma nova organização substanciada por análises fatoriais exploratórias e confirmatórias, nomeada como Escala de Urgência com o 
Saraiva, R. B., Gastal, C. A., Campelo, G. \& Iglesias, F. (2015). Escala de Urgência com o Tempo e Ocupação Constante: Adaptação, Validade e Versão Reduzida.

Tabela 3

Correlações Entre os Fatores da UTOC e Demais Variáveis

\begin{tabular}{|c|c|c|c|c|c|c|c|c|c|}
\hline & 1 & 2 & 3 & 4 & 5 & 6 & 7 & 8 & 9 \\
\hline \multicolumn{10}{|l|}{ 1. TUPA - Global } \\
\hline 2. TUPA - Urgência com o Tempo & $0,98 * *$ & - & & & & & & & \\
\hline 3. TUPA - Ativação Constante & $0,99 * *$ & $0,96 * *$ & - & & & & & & \\
\hline 4. UTOC - Urgência com o Tempo & $0,91 * *$ & $0,87 * *$ & $0,89 * *$ & - & & & & & \\
\hline 5. UTOC - Ocupação Constante & $0,74 * *$ & $0,74 * *$ & $0,75^{* *}$ & $0,46 * *$ & - & & & & \\
\hline 6. UTOC - Versão reduzida & $0,95 * *$ & $0,93 * *$ & $0,94 * *$ & $0,93 * *$ & $0,67 * *$ & - & & & \\
\hline 7. IDATE - Ansiedade Presente & $0,36 * *$ & $0,34 * *$ & $0,38 * *$ & $0,46^{* *}$ & $0,12 * *$ & $0,38 * *$ & - & & \\
\hline 8. IDATE - Ansiedade Ausente & $-0,30 * *$ & $-0,27 * *$ & $-0,31 * *$ & $-0,37 * *$ & $-0,11^{*}$ & $-0,32 * *$ & $-0,69 * *$ & - & \\
\hline 9. PSS - Estresse Percebido & $0,39 * *$ & $0,38 * *$ & $0,39 * *$ & $0,43 * *$ & $0,22 * *$ & $0,39 * *$ & $0,78 * *$ & $-0,67 * *$ & - \\
\hline 10. PSS - Coping Percebido & $-0,18 * *$ & $-0,14 * *$ & $-0,19 * *$ & $-0,30 * *$ & 0,02 & $-0,23 * *$ & $-0,68 * *$ & $0,73 * *$ & $-0,69 * *$ \\
\hline
\end{tabular}

Nota. UTOC: Escala de Urgência com o Tempo e Ocupação Constante; IDATE: Inventário de Ansiedade Traço-Estado; PSS: Escala de Estresse Percebido. ${ }^{*} p<0,05 ; * * p<0,01$.

Tempo e Ocupação Constante (UTOC), assim como uma versão reduzida.

Inicialmente cabe discutir a comparação entre a composição fatorial original da escala e a nova composição obtida neste estudo. Ambos os modelos obtiveram índices psicométricos satisfatórios. Ambos os modelos obtiveram índices psicométricos satisfatórios, porém, as duas versões apresentam notáveis distinções quanto à organização de seus fatores. Porém, as duas versões apresentam notáveis distinções quanto à organização de seus fatores. Os autores da escala original apresentam uma versão com um fator global e dois fatores específicos relacionados à urgência com o tempo e ativação constante. Já no presente estudo a configuração mais apropriada foi bifatorial, com um fator referente à urgência com o tempo e um fator de ocupação constante. É importante ressaltar que o novo fator relacionado à ocupação constante se difere conceitualmente do fator original relacionado a ativação constante, apesar de compartilharem alguns itens.

A ativação constante, tal como descrita pelos autores da TUPA, diz respeito principalmente à excessiva ativação motora, o que caracteriza indivíduos que estão constantemente procurando atividades, ou seja, que consideram aversivo permanecer parado ou não realizar uma atividade por muito tempo (ex: "Quando preciso ficar sentado, eu manuseio um objeto - como um lápis, faço movimentos com os dedos, movimento meus dentes, ou não fico completamente parado"; Wright et al., 1992). Já o novo fator trata mais especificamente de aspectos relacionados à quantidade de compromissos e tarefas às quais um indivíduo se submete (ex: "Eu geralmente tenho dias extremamente cheios"). Evidências para a distinção de tais fatores podem ser observadas em seus índices de correlação com a IDATE e a PSS. Na presente análise a Ativação Constante aparece positivamente relacionada com fatores de ansiedade e estresse, enquanto estas mesmas relações são atenuadas ou até mesmo desaparecem no fator de Ocupação Constante. Sendo assim a Ocupação Constante pode ser interpretada como um fator que acessa a quantidade de compromissos de forma objetiva, não sendo necessariamente associada a fatores estressantes. Já a Urgência com o Tempo e a Ativação Constante possuem uma associação mais evidente com o estresse e a ansiedade, sinalizando situações em que a rotina acelerada de indivíduos passa a ter efeitos negativos (Conte et al., 1998; Landy et al., 1991).

É possível considerar motivos tanto culturais quanto metodológicos para explicar a obtenção de uma composição fatorial diferente no Brasil. Além de diferenças no tamanho e na caracterização da amostra neste trabalho e do original da escala, considera-se que a maneira como indivíduos lidam com o tempo é um aspecto que possui grande diversidade cultural (Levine \& Norenzayan, 1999; Sircova et al., 2014). Existem evidências, por exemplo, que a percepção temporal de brasileiros é bem distinta dos estado-unidenses no que se refere a pontualidade (Levine, West, \& Reis, 1980). Ambas as versões apresentaram índices psicométricos que as tornam aptas para o uso, porém, recomenda-se a utilização da nova composição bifatorial (UTOC). Um aspecto relevante para a utilização da UTOC é que, apesar dos fatores serem estreitamente relacionados, são facilmente diferenciáveis e bem definidos. Não existem itens comuns entre os dois fatores, o que facilita a definição de cada construto e a 
compreensão de suas relações com outras variáveis. $\mathrm{O}$ mesmo não ocorre na TUPA original, em que grande parte dos itens são compartilhados entre os fatores. Em relação à análise fatorial confirmatória, a UTOC apresentou índices adequados. Apesar de não apresentar a mesma composição da TUPA, limitando comparações diretas, o uso de novas composições fatoriais está de acordo com as diretrizes da American Educational Research Association, da American Psychological Association $e$ do National Council on Measurement in Education (1999), que prevêem a atualização e aperfeiçoamento de instrumentos, considerando época e cultura da população que recebe a avaliação psicológica.

Quanto à validação convergente, as relações obtidas entre a UTOC e as demais variáveis exploradas neste estudo mostraram-se em consonância com as relações já estabelecidas pela literatura. Os principais resultados foram correlações positivas dos fatores da UTOC com Estresse Percebido e Ansiedade Presente, assim como correlações negativas com os fatores Coping Percebido e Ansiedade Ausente. Tais resultados estão de acordo com outros estudos que encontraram maiores níveis de stress e ansiedade em indivíduos mais ocupados e preocupados com seu tempo (Conte et al., 2001; Menon et al., 2013).

A presente pesquisa apresenta algumas limitações. A primeira diz respeito ao tamanho e caracterização da amostra, que não pode ser considerada representativa do Brasil. Contudo, considera-se que a quantidade de participantes foi suficiente para conhecer os parâmetros psicométricos da UTOC. É importante ressaltar também a supressão de seis itens da escala original, referentes à comportamento no trânsito. Tal decisão impossibilitou a avaliação de cargas fatoriais e contribuições psicométricas desses itens, além de diminuir a viabilidade de comparações vis à vis dos resultados obtidos com a UTOC brasileira e a versão original. Por outro lado, a exclusão de tais itens teve por objetivo conferir maior aplicabilidade à escala, uma vez que não é mais exigido que os respondentes tenham quaisquer experiências relacionadas ao trânsito. Uma consideração importante deve ser feita também sobre o uso de análise fatorial exploratória e análise fatorial confirmatória com a mesma amostra. Embora Van Prooijen e Van der Kloot (2001) tenham mostrado até mesmo algumas vantagens metodológicas desse tipo de estratégia, ela pode enviesar os índices de adequação dos modelos testados, superestimando os resultados da análise confirmatória e prejudicando interpretações definitivas. Sendo assim, resta ainda testar a composição e a consistência da escala em diferentes contextos (idealmente com diferentes métodos, como sugerem Hurley et al., 1997), assim como a estabilidade de suas propriedades psicométricas ao longo do tempo.

Em suma, o objetivo de adaptação de uma escala de urgência com o tempo foi alcançado, com evidências complementares de validade, dando origem a três versões do instrumento, todas com propriedades psicométricas sólidas. Os índices de consistência interna da escala no presente estudo foram ainda mais robustos que os obtidos no estudo original (Wright et al., 1992). Espera-se, desta forma, que a UTOC contribua em uma área que possui grandes limitações quanto a disponibilidade de instrumentos de medida, como mais um recurso relevante para a investigação do papel de variáveis temporais em comportamentos e atitudes. Além de uma alternativa para investigações empíricas, a escala também possui amplo potencial de aplicabilidade prática. Em contextos hospitalares e clínicos a escala pode ser utilizada para mapear níveis patológicos de estresse e ansiedade provenientes da preocupação excessiva com o tempo, assim como prever tendências à doenças coronárias e outras enfermidades (Conte et al., 1998; Shi et al., 2013). O papel de variáveis temporais também é muito enfatizado nas relações entre felicidade e dinheiro que são tipicamente investigadas na economia comportamental (Aaker, Rudd, \& Mogilner, 2011). Revisões da área também apontam que em ambientes organizacionais a urgência com o tempo é uma relevante preditora de desempenho, tendo em vista suas relações com estresse e ansiedade (Conte et al., 2001; Greenberg, 2002; Menon et al., 2013). Para tais contextos em que muitas vezes a aplicação de instrumentos deve ser rápida e econômica, propôs-se uma versão reduzida com os itens mais representativos da escala, considerando-se obviamente as suas vantagens e desvantagens frente à versão completa do instrumento.

\section{Referências}

Aaker, J., Rudd, M., \& Mogilner, C. (2011). If money doesn't make you happier, consider time. Journal of Consumer Psychology, 21, 126-130. doi:10.2139/ssrn.1706968

Amelang, M. (1997). Using personality variables to predict cancer and heart disease. European Journal of Personality, 11(5), 319-342. doi:10.1002/(SICI)1099-0984(199712)11:5<319::AID-PER304>3.0.CO;2-D

American Educational Research Association, American Psychological Association, \& National Council on Measurement in Education. (1999). Standards for educational and psychological testing. Washington, DC: American Educational Research Association.

Biaggio, A. M. B., \& Natalício, L. (1979). Manual para o Inventário de Ansiedade Traço-Estado (IDATE). Rio de Janeiro, RJ: Centro Editor de Psicologia Aplicada.

Bluedorn, A. C., Kaufman, C. F., \& Lane, P. M. (1992). How many things do you like to do at once? An introduction to monochronic and polychronic time. Academy of Management Executive, 6(4), 17-26. doi:10.5465/AME.1992.4274453

Boomsma, A. (2000). Reporting analyses of covariance structures. Structural Equation Modeling, 7(3), 461-483. doi:10.1207/S15328007SEM0703_6

Britton, B. K., \& Tesser, A. (1991). Effects of time-management practices on college grades. Journal of Educational Psychology, 83, 405-410. doi:10.1037/0022-0663.83.3.405

Byrne, B. (2010). Structural equation modeling with Amos: Basic concepts, applications and programming. New York: Taylor and Francis.

Claessens, B. J., van Eerde, W., Rutte, C. G., \& Roe, R. A. (2007). A review of the time management literature. Personnel Review, 36(2), 255-276. doi:10.1108/00483480710726136 
Saraiva, R. B., Gastal, C. A., Campelo, G. \& Iglesias, F. (2015). Escala de Urgência com o Tempo e Ocupação Constante: Adaptação, Validade e Versão Reduzida.

Cohen, S., Karmack, T., \& Mermelsteinm, R. (1983). A global measure of perceived stress. Journal of Health and Social Behavior, 24(4), 385-396.

Conte, J. M., Landy, F. J., \& Mathieu, J. E. (1995). Time urgency: Conceptual and construct development. Journal of Applied Psychology, 80, 178-185. doi:10.1037/0021-9010.80.1.178

Conte, J. M., Mathieu, J. E., \& Landy, F. J. (1998). The nomological and predictive validity of time urgency. Journal of Organizational Behavior, 19, 1-13. doi:10.1002/(SICI)1099-1379(199801)19:1<1::AID-JOB815>3.0.CO;2-E

Conte, J. M., Schwenneker, H. H., Dew, A. F., \& Romano, D. M. (2001). Incremental validity of time urgency and other Type A subcomponents in predicting behavioral and health criteria. Journal of Applied Social Psychology, 31(8), 17271748. doi:10.1111/j.1559-1816.2001.tb02748.x

DeVoe, S. E., \& House, J. (2012). Time, money, and happiness: How does putting a price on time affect our ability to smell the roses? Journal of Experimental Social Psychology, 48, 466-472. doi:10.1016/j.jesp.2011.11.012

Edwards, J. R., Baglioni, A. J., Jr., \& Cooper, C. L. (1990). Examining relationships among self-report measures of Type A behavior pattern: The effects of dimensionality, measurement error, and differences in underlying constructs. Journal of Applied Psychology, 75, 440-454. doi:10.1037/00219010.75.4.440

Fernandes, R., Hatfield, J., \& Soames Job, R. F. (2010). A systematic investigation of the differential predictors for speeding, drink-driving, driving while fatigued, and not wearing a seat belt, among young drivers. Transportation Research Part F: Traffic Psychology and Behaviour, 13(3), 179-196. doi:10.1016/j.trf.2010.04.007

Fioravanti, A. C., Santos, L. F., Cruz, A. P. M., \& Landeira-Fernandez, J. (2006). Avaliação da estrutura fatorial da escala de ansiedade-traço do IDATE. Avaliação Psicológica, $5,217-224$

Friedman, M., \& Rosenman, R. H. (1959). Association of specific overt behavior pattern with blood and cardiovascular findings. The Journal of the American Medical Association, 169, 1286 1296. doi:10.1001/jama.1959.03000290012005

Friend, K. E. (1982). Stress and performance: Effects of subjective work load and time urgency. Personnel Psychology, 35, 623-633. doi:10.1111/j.1744-6570.1982.tb02214.x

Gastorf, J. W. (1980). Time urgency of the Type A behavior pattern. Journal of Clinical and Consulting Psychology, 48, 299. doi:10.1111/j.1744-6570.1982.tb02214.x

Glass, D. C., Snyder, M. L., \& Hollis, J. F. (1974). Time urgency and the Type A coronary-prone behavior pattern1. Journal of Applied Social Psychology, 4(2), 125-140. doi:10.1111/j.1559-1816.1974.tb00663.x

Greenberg, J. (2002). Time urgency and job performance: Field evidence of an interactionist perspective. Journal of Applied Social Psychology, 32(9), 1964-1973. doi:10.1111/j.1559-1816.2002.tb00267.x

Hurley, A. E., Scandura, T. A., Schriesheim, C. A., Brannick, M. T., Seers, A., Vandenberg, R. J., \& Williams, L. J. (1997). Exploratory and confirmatory factor analysis: Guidelines, issues, and alternatives. Journal of Organizational Behavior, 18, 667683. doi:10.1002/(SICI)1099-1379(199711)18:6<667::AIDJOB874>3.0.CO;2-T

Jenkins, C. D., Zyzanski, S. J., \& Rosenman, R. H. (1979). Jenkins Activity Survey Manual (Form C). New York: Psychological Corp.
Kohler, S. S. (1991). Time urgency: Psychophysiological correlates (Unpublished doctoral dissertation). University Park, Pennsylvania State University, State College, PA.

Krantz, D. S., Glass, D. C., \& Snyder, M. L. (1974). Helplessness, stress level, and the coronary-prone behavior pattern. Journal of Experimental Social Psychology, 10, 284-300. doi:10.1016/0022-1031(74)90074-2

Landy, F. J., Rastegary, H., Thayer, J., \& Colvin, C. (1991). Time urgency: The construct and its measurement. Journal of Applied Psychology, 76, 644-657. doi:10.1037/00219010.76.5.644

Leite, U. R., \& Pasquali, L. (2008). Estudo de validação do Inventário de Perspectiva de Tempo do Zimbardo. Avaliação Psicológica, 7(3), 301-320.

Levine, R. V., \& Norenzayan, A. (1999). The pace of life in 31 countries. Journal of Cross-Cultural Psychology, 30, 178-205. doi: $10.1177 / 0022022199030002003$

Levine, R. V., West, L. J., \& Reis, H. T. (1980). Perceptions of time and punctuality in the United States and Brazil. Journal of Personality and Social Psychology, 38(4), 541-550. doi:10.1037/0022-3514.38.4.541

Luft, C. D. B., Sanches, S. O., Mazo, G. Z., \& Andrade, A. (2007). Versão brasileira da Escala de Estresse Percebido: Tradução e validação para idosos. Revista de Saúde Pública, 41(4), 606-615. doi:10.1590/S0034-89102007000400015

Macan, T. H., Shahani, C., Dipboye, R. L., \& Phillips, A. P. (1990). College students time management: Correlations with academic performance and stress. Journal of Educational Psychology, 82, 760-768. doi:10.1037/0022-0663.82.4.760

Mardia, K. V. (1971). The effect of nonnormality on some multivariate tests and robustness to nonnormality in the linear model. Biometrika, 88(1), 105-121. doi:10.1093/ biomet $/ 58.1 .105$

McDonald, R. P., \& Ho, M. R. (2002). Principles and practice in reporting structural equation analyses. Psychological Methods, 7(1), 64-82. doi:10.1037/1082-989X.7.1.64

Menon, S., Narayanan, L., \& Spector, P. E. (2013). Time urgency and its relation to occupational stressors and health outcomes for health care professionals. In C. D. Spielberger \& I. G. Sarason (Eds.), Stress and emotion: Anxiety, anger and curiosity (pp. 127-142). Washington, DC: Taylor and Francis.

Milfont, T. L., Andrade, P. R., Pessoa, V. S., \& Belo, R. P. (2008). Testing Zimbardo Time Perspective Inventory in a Brazilian sample. Interamerican Journal of Psychology, 42(1), 49-58.

Rosenman, R. H., Friedman, M., Straus, R., Wurm, M., Jenkins, C. D., \& Messinger, H. B. (1966). Coronary heart disease in the Western collaborative group study: A follow-up experience of two years. Journal of the American Medical Association, 195, 130-136. doi:10.1001/jama.1966.03100020074017

Sircova, A., van de Vijver, F. J. R., Osin, E., Milfont, T. L., Fieulaine, N., Kislali-Erginbilgic, A., ...Boyd, J. N. (2014). A global look at time: A 24-country study of the equivalence of the Zimbardo Time Perspective Inventory. SAGE Open, 4(1), 1-12. doi:10.1177/2158244013515686

Sirri, L., Fava, G. A., Guidi, J., Porcelli, P., Rafanelli, C., Bellomo, A., ...Sonino, N. (2012). Type A behaviour: A reappraisal of its characteristics in cardiovascular disease. International Journal of Clinical Practice, 66(9), 854-861. doi:10.1111/j.1742-1241.2012.02993.x 
Shi, H., Yang, X., Wang, J., Xi, H., Huang, C., He, J., ...Zhuang, G. (2013). Type A personality, hostility, time urgency and unintentional injuries among Chinese undergraduates: A matched case-control study. BMC Public Health, 13(1066), 1-10. doi:10.1186/1471-2458-13-1066

Spielberger, C. D., Gorsuch, R. L., \& Lushene, R. D. (1970). STAI: Manual for the State-Trait Anxiety Inventory. Palo Alto, CA: Consulting Psychologists Press.

Tabachnick, B., \& Fidell, L. S. (2013). Using multivariate statistics. Upper Saddle River, NJ: Pearson.

Usunier, J. G., \& Valette-Florence, P. (1994). Perceptual time patterns ('time styles'): A psychometric scale. Time \& Society, 3(2), 219-241. doi:10.1177/0961463X94003002005

Van Prooijen, J. W., \& Van Der Kloot, W. A. (2001). Confirmatory analysis of exploratively obtained factor structures. Educational and Psychological Measurement, 61(5), 777-792. doi:10.1177/00131640121971518

Van de Vijver, F. J. R., \& Leung, K. (2011). Equivalence and bias: A review of concepts, models, and data analytic procedures. In D. Matsumoto \& F. J. R. van de Vijver (Eds.), Cross-cultural research methods in psychology (pp. 17-45). New York: Cambridge University Press.

Wright, L., McCurdy, S., \& Rogoll, G. (1992). The TUPA scale: A self-report measure for the type A subcomponent of time urgency and perpetual activation. Psychological Assessment, 4(3), 352-356. doi:10.1037/1040-3590.4.3.352

Wright, L., Nielsen, B. A., Abbanato, K. R., Jackson, T., Lancaster, C., \& Son, J. (1995). The relationship of various measures of time urgency to indices of physical health. Journal of Clinical Psychology, 51(5), 610-614. doi:10.1002/10974679(199509)51:5<610::AID-JCLP2270510505>3.0.CO;2-3

Wright, L., \& Schmidt-Walker, K. (1990). An augmented structured interview for measuring subcomponents of the Type A behavior pattern. International Journal of Psychiatry in Medicine, 20, 85-96. doi:10.2190/YDK9-J9NV-5RA2-RXDV

Yarnold, P. R., \& Grimm, L. G. (1982). Time urgency among coronary-prone individuals. Journal of Abnormal Psychology, 91, 175-177. doi:10.1037/0021-843X.91.3.175

Yarnold, P. R., \& Mueser, K. T. (1984). Time urgency of Type A individuals: Two replications. Perceptual and Motor Skills, 58, 334. doi:10.2466/pms.1984.59.1.334

Yousfi, S., Matthews, G., Amelang, M., \& Schmidt-Rathjens, C. (2004). Personality and disease: Correlations of multiple trait scores with various illnesses. Journal of Health Psychology, 9(5), 627-647. doi:10.1177/1359105304045339

Zimbardo, P. G., Keough, K. A., \& Boyd, J. N. (1997). Present time perspective as a predictor of risky driving. Journal of Personality and Individual Differences, 23, 1007-1023. doi:10.1016/S0191-8869(97)00113-X 